\section{Expert Opinion}

1. Introduction

2. TCR gene transfer

3. T-bodies

4. Clinical trials

5. Expert opinion

\title{
Re-targeting T-cells against cancer by gene-transfer of tumor-reactive receptors
}

\author{
Victoria Marcu-Malina, Suzanne van Dorp \& Jürgen Kuball ${ }^{\dagger}$ \\ ${ }^{\dagger}$ University Medical Center Utrecht, Departments of Immunology and Hematology and VanCreveld Clinic, \\ Huispostnr: KC02.085.2; Kamer KB.02.043.1, Lundlaan 6, 3584 EA Utrecht, The Netherlands
}

\begin{abstract}
Background: Adoptive transfer of T-lymphocytes is a promising treatment for a variety of malignancies, but is often not feasible due to difficulties in generating T-cells reactive with the targeted antigen from patients. To facilitate rapid generation of cells for therapy, T-cells can be programmed with genes encoding for an antigen-specific T-cell receptor (TCR) or chimeric receptors. Objective: To discuss the molecular design and selected pitfalls of TCR gene modified T-cells and T-cells expressing chimeric receptors, so called T-bodies. Methods: A selected review of the recent literature. Conclusion: Clinical trials report so far only limited efficacy of adoptively transferred genetically modified T-cells. However, the recent progress in engineering tumor-reactive $T$ cells is providing a promising basis to further explore this treatment modality.
\end{abstract}

Keywords: clinical trial, genetic engineering, T-body, TCR gene transfer

Expert Opin. Biol. Ther. (2009) 9(5):579-591

\section{Introduction}

The application of molecular technologies to identify proteins differentially expressed by transformed cells is providing large numbers of candidate antigens that can potentially be targeted to selectively eliminate tumor cells by cancer immunotherapy [1,2]. Efforts to vaccinate patients against such antigens have yielded some provocative results, but only a small subset of patients have demonstrated therapeutic responses, probably reflecting the many in vivo obstacles to generating potent responses to these proteins, particularly in patients with an established malignancy [3]. An alternative approach of isolating and expanding reactive T-cells ex vivo followed by adoptive transfer into the patient circumvents many of these in vivo obstacles. Although this adoptive therapy approach has demonstrated significant clinical promise [4], generating the large numbers of T-cells required for adoptive therapy of cancer patients is often not feasible. Molecular technologies have now provided a means to more broadly capture the therapeutic potential of this treatment strategy (Figure 1). Genes encoding the $\alpha$ and $\beta$ chains of a T-cell receptor (TCR) can be isolated from a T-cell reactive to the antigen of interest and restricted to a defined HLA allele, inserted into a shuttle expression vector, and then introduced into large numbers of T-cells of individual patients sharing the restricting allele and the targeted protein [5,6]. Alternatively a T-body approach is pursued, which combines benefits of the high tumor specificity of antibodies and the effector function and the homing abilities of T-cells. The chimeric receptor recognition unit is derived from the variable chain of an antibody recognizing proteins ideally selectively expressed at the cell surface of a cancer cell and the intracellular domain is usually composed of signaling 


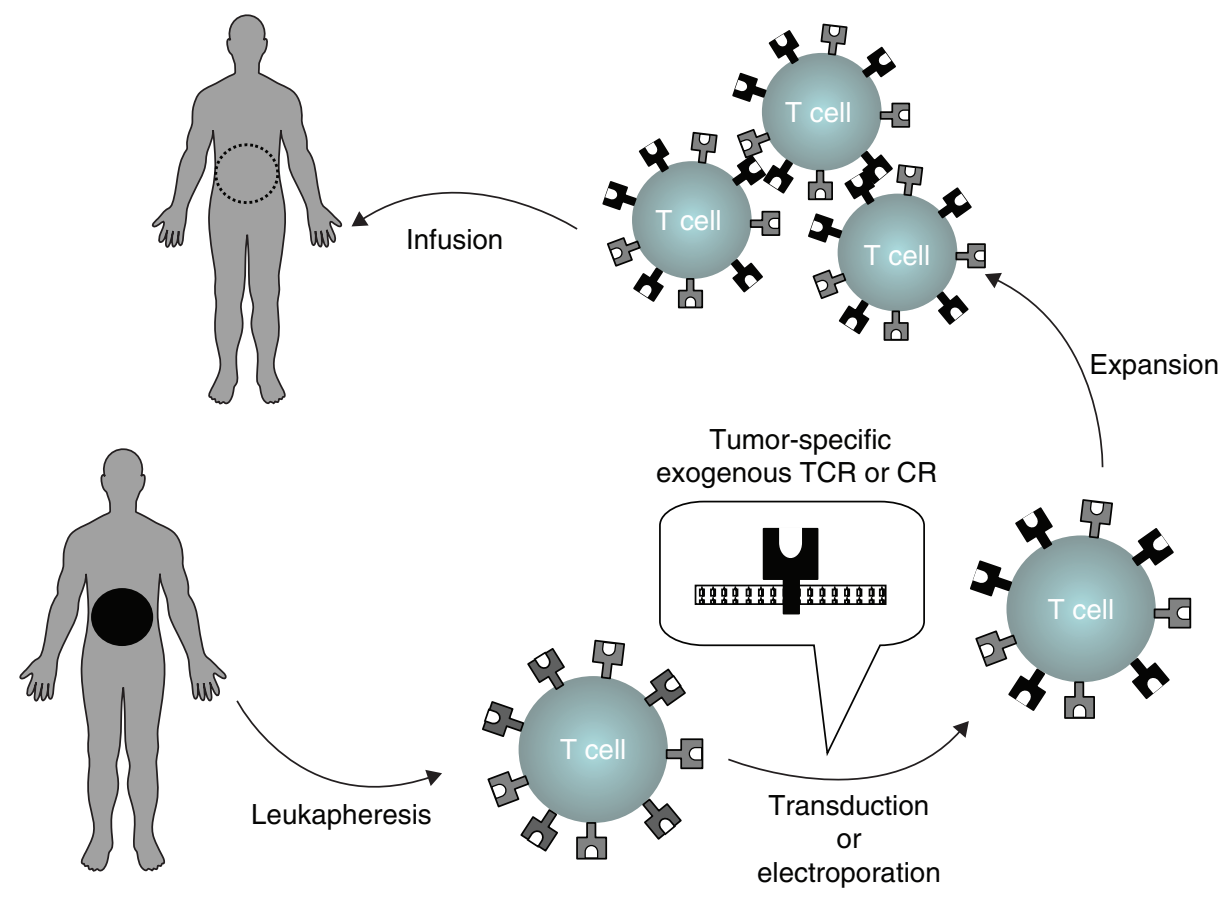

Figure 1. Adoptive transfer of genetically modified T cells. T cells isolated from a patient are genetically modified by, for example, retro-/lentiviral transduction or electroporation to express a tumor-specific receptor. After in vitro expansion cells are infused back into the patient.

CR: chimeric receptor; TCR: T cell receptor.

and co-signaling moieties of a T-cell receptor [7]. This review focuses on selected challenges and pitfalls of these strategies and published or ongoing clinical trials.

\section{TCR gene transfer}

Multiple virus- and tumor-reactive TCR-genes have already been successfully isolated and re-expressed in T-cells, including TCR-genes with specificity for HLA*0201 (HLA-A2)restricted epitopes from melanoma antigens $[8,9]$ and HLA-A2 and HLA*2402-restricted WT1-derived epitopes [5,10] However, to design broadly tumor-reactive TCRs for the next generation of clinical TCR gene therapy efforts, several aspects have to be taken into account. The TCR should target a protein that is widely expressed in tumors of any origin and cover a range of frequent HLA types. Furthermore, the targeted protein should be inherently involved in tumor transformation and selectively expressed by tumors and not by normal tissue. The TCR must be chosen for appropriate affinity for its ligand, be expressed strongly in T-cells after transfer and consequently allow reprogramming of $\mathrm{CD}^{+}$helper/effector and $\mathrm{CD}^{+}$effector T-cells. The design of introduced TCR chains must prevent pairing of introduced TCR chains with endogenous ones. This minimizes the generation of new potentially auto-reactive TCR specificities and simultaneously increases T-cell avidity by increasing the amount of functional TCR chains at the cell surface.

\subsection{Engineering TCRs with high affinity against tumor antigens}

A major challenge of current TCR gene transfer strategies is to define means to improve the avidity of T-cells transduced with a potentially tumor-reactive TCR, particularly in the context of the generally low affinity of available tumor-reactive TCRs. Therefore it is desirable to select tumor-reactive TCRs with the highest identifiable affinity [10-12] and explore strategies to increase TCR-affinity before transduction $[13,14]$ or the (functional) avidity of the resulting TCR-transduced T-cells [5,15-17]. In this context, 'affinity' will be defined as the strength of binding of one receptor with its ligand, 'avidity' as strength of binding between multiple receptors and their ligands, and 'functional avidity' as the sensitivity of a T-cell response to a target cell expressing the relevant peptide-MHCs (pMHCs) [18].

Several approaches have been reported to select TCRs with highest affinity from the human T-cell repertoire. For example, alpha 3 domain mutants of peptide/MHC class I multimers have been used to allow the selective isolation of high avidity tumor-reactive $\mathrm{CD} 8^{+} \mathrm{T}$-cells [19]. To overcome the TCR affinity threshold determined by thymic selection, tumor-antigen-specific HLA-A*0201-restricted T-cells have been generated from HLA-A*0201 negative patients $[11,20]$. HLA-A*0201-transgenic mouse models [12] can, due to the differences in protein sequences in mice and men, provide TCRs with such a high affinity that these TCRs can also activate T-cells in the absence of the co-stimulatory CD8 
molecule. However, expressing mouse TCRs in human T-cells might have a disadvantage as the expression of altered molecules results in a T-cell response against the transgene and rejection of transduced T-cells [21].

Different strategies have been examined to further increase the affinity of cloned TCRs used for transduction. TCR-phage [14] and -yeast [13] displays have been used as formats to express TCR chains and to then generate mutants that can be screened ex vivo for increased affinity. With these approaches, a library of TCR chains can be created generally following random mutation of defined regions such as CDR3 known to be important in recognition; the library expressed on the surface of the phage or yeast; the expressed mutated TCRchains screened by tetramer-binding for increased affinity; and the cDNA encoding the highest affinity chains extracted, characterized and subsequently used for transduction. Consequently, these display strategies can be very effective, but are cumbersome and must be individualized for each TCR. Furthermore, not every TCR can be successfully expressed and modified by these technically challenging display strategies in non-mammalian systems, emphasizing the need for alternative techniques to increase TCR-affinity or functional avidity of a T-cell [18].

The functional avidity of a T-cell, as reflected by responsiveness to antigen, is modulated by T-cell surface $O$-or $N$-glycosylation [22,23]. Decreased glycosylation of surface proteins, has been reported to result in a decreased activation threshold. For example, decreased glycosylation of CD8 during thymic development is associated with increased affinity of CD8 with pMHC and subsequently improved signaling [24]. Moreover, a general deficiency in $\beta 1,6 \mathrm{~N}$-acetylglucosaminyltransferase V (Mgat5) in mice, an enzyme in the $N$-glycosylation pathway, mediates increased T-cell activity in vitro and results in autoimmune disease in vivo [25]. Although deficiency of this $N$-glycosylation pathway enzyme in mice resulted in reduced $N$-glycosylation of all proteins, the reduced $N$-glycosylation of the TCRs appeared to specifically result in increased TCR mobility at the cell surface, enhanced recruitment to the synapse (TCR-clustering), improved TCRengagement/downmodulation, and consequently enhanced functional avidity of T-cells. We demonstrated recently with several TCRs recognizing different antigens that the removal by point mutation of defined $N$-glycosylation motifs in the constant domains of TCR-chains can increase the functional avidity of T-cells transduced with these TCRs, and that this translates into enhanced recognition of tumor cells. Reducing $N$-glycosylation of TCRs might thereby directly enhance the interaction of TCRs with pMHCs and consequently increase T-cell activation [18]. As these $N$-glycosylation sites in the constant domain are conserved in all TCRs, this strategy should be easily translated to TCRs with any specificity.

An undesirable effect of an increased avidity of a tumorantigen-specific T-cell might be the recognition of normal tissue either by aberrant recognition of self-antigens [26] or recognition of the specifically targeted tumor antigen, such as Wilms tumor 1 (WT1) [27], p53 [28], or murine double minute 2 homolog (MDM2) [11], due to the physiological expression of the protein at lower levels in normal tissues. It has indeed been reported that increasing the TCR affinity above a certain threshold can result in the recognition of self peptide and therefore auto-reactivity [26]. Therefore, high affinity TCRs need to be tested thoroughly for the 'recognition of self' [18].

\subsection{TCR matching at the cell surface}

Even after selecting a TCR with the highest affinity, the introduced exogenous $\alpha$ and $\beta$ chains can potentially assemble as pairs not only with each other but with the endogenous TCR $\alpha$ and $\beta$ chains, thereby reducing the number of appropriately matched exogenous $\alpha \beta$ TCR-pairs at the cell surface and decreasing the achievable functional T-cell avidity (Figure 2). Such mismatched pairing poses a second substantive problem for clinically pursuing this strategy - the generation of novel $\alpha \beta$ TCR-pairs of undefined and potentially self-reactive specificity, as these TCRs have not been subjected to the normal rigors of negative selection. Mismatched pairing of $\alpha \beta$ TCR-chains has been clearly demonstrated to occur in TCR double-transgenic mice [29], in which all four of the expressed TCR-chains are known and can be followed. Since export of TCR-chains to the cell surface only occurs after formation of complementary $\alpha$ and $\beta$ subunits [30], the potential for pairing of introduced TCR-chains with endogenous TCR-chains has also been demonstrated by introducing only single $\alpha$ or $\beta$ TCR-chains into murine [31] and human [32] T-cells and detecting expression of the introduced chain on the cell surface. Unpublished data from different laboratories suggest that this can be indeed a substantial problem for adoptive immunotherapy.

To promote preferential pairing of introduced TCR-chains with each other, several strategies might be pursued (Figure 2). Recently, we [11,33] and others [15] have been using murine constant TCR-chain domains to enhance expression of human TCR-chains in human T-cells. However, murine constant domains might be immunogeneic in patients, thereby limiting the survival of transgene-transduced T-cells in patients [34]. The intracellular TCR-chain domains can also be linked to a signaling domain and this has been reported to result in CD3-independent signaling of TCR transduced T-cells [35]. However, as modulating the transmembrane domain might also result in unwanted effects such as an altered T-cell function [36] alternative approaches are being explored. Interactions between the extracellular domains, which can contribute to the formation of individual TCR-dimers and CD3-complexes [37,38], might be modified to facilitate inter-chain attraction. The interactions between exogenous domains of the TCR $\alpha$ and $\beta$ chains are generally very weak [39], and the unique naturally occurring inter-chain disulfide-bond that occurs between the constant TCR $\alpha$ and $\beta$ chains does not appear to contribute significantly to heterodimer stability [40]. However, disulfidebonds engineered in other sites in the extracellular constant domain of the TCR $\alpha \beta$-heterodimer may have the potential 

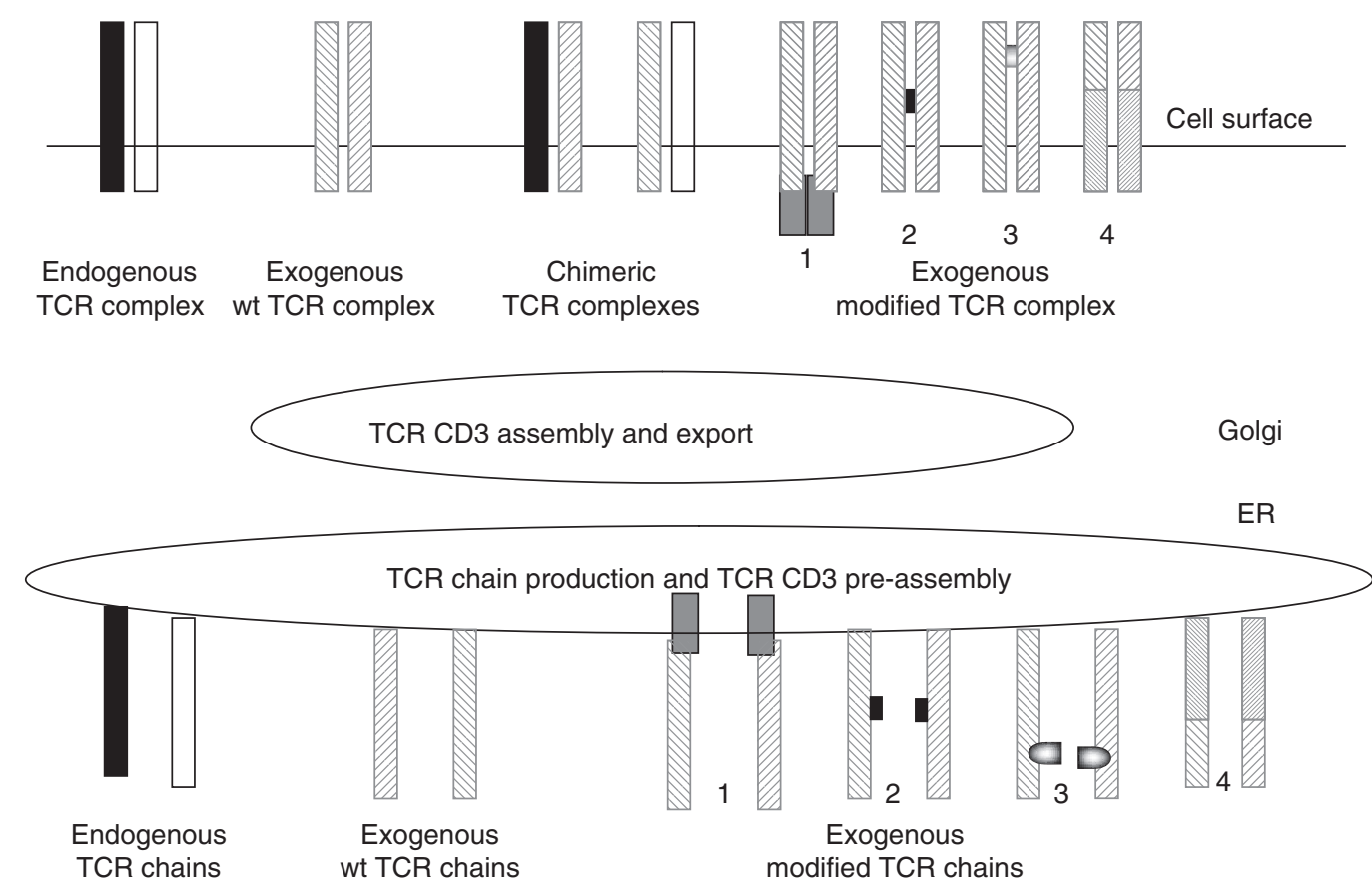

Figure 2. Improving matched TCR $\alpha \beta$ chain assembly of introduced TCR chains. Introduced and endogenous $\alpha$ and $\beta$ TCR chains can pair with each other. This creates TCRs of unwanted specificity and reduces the total number of correctly matched TCR chains at the cell surface. To promote pairing of the introduced chains genetic modifications can be made, such as 1 - addition of an intracellular signaling domain derived from $\mathrm{CD} 3 \zeta ; 2$ - addition of cysteines or mutations to gain the 'hole-into-knob configuration' in the extracellular constant domains; 3 - selection of naturally occurring variable chains that preferentially pair with each other or generation of preferentially paired variable TCR chains by mutagenesis; and 4 - murinization of the constant $\alpha \beta$ TCR chains.

to increase inter-chain affinity, as suggested by such disulfidebonds enhancing formation of soluble TCR-heterodimers [41]. We found [5] and others confirmed [42] that such point mutations both promoted preferential pairing and increased expression of the introduced chains, resulting in greater avidity of the transduced primary CD8 ${ }^{+}$T-cells for WT1-expressing targets. Alternatively or additionally, a pair of amino acid residues in the crystal structure of a TCR that lie at the interface of associated TCR constant $\alpha$ and $\beta$ domains can be mutated to invert the sense of this interaction analogous to a charged 'hole-into-knob' configuration [43]. Finally, also structural features of the variable TCR regions might be used to promote preferential pairing either by selecting TCRs with variable chains that pair preferentially to each other [44] or by introducing point mutations in the variable domain as reported for the expression of single-chain variable $\alpha$ and $\beta$ fragments [45]. Although not formally tested so far, a combination of these different strategies might provide the best results in terms of preventing mismatched pairing.

\subsection{Improving expansion and memory formation of TCR transduced T-cells}

An antigen-specific T-cell expansion is one of the hallmarks for control of viral infections [46] and tumor growth [47]. Thus strategies that would allow to improve the expansion phase during an anti-tumor immune response would increase efficacy of immunotherapies. Therefore, the transgene should be incorporated into central memory T-cells in order to achieve a long term persistence of transduced T-cells [48]. This can for example be achieved by transducing and expanding genetically modified T-cells in the presence of cytokines such as IL7 and IL15 [49]. Detectable expansion of EBV-reactive $\mathrm{T}$ cells is found uniformly after $\mathrm{T}$ cell transfer into lymphopenic patients after hematopoetic stem cell transplantation [50], and consequently lymphodepletion has been successfully explored to improve T-cell expansion and clinical response rates following adoptive transfer of tumor-specific T-cells [47]. Elimination of $\mathrm{CD}^{+}{ }^{+} \mathrm{CD} 25^{+}$regulatory T-cells has been proposed as a key mechanism by which lymphodepletion augments adoptive T-cell-transfer-based immunotherapy [51]. A recent report suggested also that lymphodepletion removes endogenous cellular elements that act as sinks for cytokines capable of augmenting the activity of self/tumor-reactive $\mathrm{CD} 8^{+}$ T-cells [52]. Nevertheless, an expansion of tumor-antigen-specific T-cells has been observed after adoptive transfer only in some patients following lymphodepletion [4,53]. Thus, although current strategies help to increase T-cell expansion following adoptive T-cell transfer, improving the intrinsic capacity of a tumor-antigen-specific T-cell to expand more extensively following antigen-encounter would be further desirable.

An improved expansion of T-cells is a result of a reduced activation induced cell death (AICD) and/or an increased 
cell division following antigen encounter. Two different TCR $\beta$ chain alterations have been reported to impair Fas ligand (FasL) secretion while maintaining other effector functions, and thus should be candidates to reduce AICD and increase consequently T-cell expansion: A fusion of the transmembrane $\gamma$ chain with a $\beta$ chain $\left(\beta_{\text {IIIchim }}\right)$ [54,55] and mutating the tyrosine $_{155}\left(\mathrm{Y}_{155}\right)$ to leucine $(\mathrm{L})$ in the trans-membrane domaine $\left(\beta_{Y 155 / L}\right)$ [56,57]. However, a potential drawback of such mutation could be that the T-cell memory formation might be impaired after extensive expansion and indeed, a recent report suggests that alterations in this transmembrane region of the $\beta$ TCR chain reduces the ability of a T-cell to build up a memory T-cell pool [36].

\section{T-bodies}

The T-body approach combines advantages of the high tumor specificity of antibodies and effector function and the homing abilities of T-cells by redirecting leukocytes with chimeric receptors whose recognition unit is derived from a variable chain of an antibody and the intracellular domain is composed from the signaling and co-signaling moiety of a TCR. Normally, a chimeric receptor is designed as a modular single chain molecule, with various structural and functional domains that allow to balance T-cell specificity and control the activation of a transduced T-cell (Figure 3) [7].

\subsection{Specificity of T-bodies}

The antibody-derived recognition unit is usually derived from the variable fragments $(\mathrm{Fv})$ of the heavy and the light chains of a given antibody that are connected via a linker to form the single chain $\mathrm{Fv}(\mathrm{scFv})$. Such receptors allow the recognition of antigen in a MHC-independent manner and in the natural appearance of antigen at the cells surface. A wide variety of monoclonal antibodies against tumor-associated-antigens has been used to engineer T-bodies. For example, several groups used diverse antibodies against human EGF receptor 2 (HER2) [58-63], which is commonly overexpressed on breast-, prostate-, kidney-cancer, and many other tumors. Other popular antigens for T-bodies are B-cell markers, such as CD19 [64-66] and CD20 [67-69], which are associated with various B-cell-derived lymphomas. Optionally, using the modularity of the chimeric receptor, the recognition unit can get access to a ligand-binding domain of a heterologous receptor (linked to the TCR signaling moiety). One example is the extracellular domain of the CD4 molecule that binds gp120, an HIV envelope protein. Such T-bodies have been reported to be efficient against HIV-infected cells [70,71].

\subsection{Modulating signaling in T-bodies}

The $\mathrm{scFv}_{\mathrm{F}}$ is linked through an extracellular linker to the transmembrane and cytoplasmic domains of lymphocyte triggering moieties such as the TCR/CD3 complex-associated $\zeta$ chain or Fc receptor $\gamma$ chain [72-74]. Upon ligand binding, these domains signal via phosphorylation of immunoreceptor tyrosine-based activation motifs (ITAMs) and subsequently activate downstream kinases from the Src family. Alternatively, those down stream tyrosine kinases such as zeta-chain (TCR) associated protein kinase $70 \mathrm{kDa}$ (ZAP70) or spleen tyrosine kinase (Syk) can serve as a signaling moiety in the chimeric receptor and lead to an efficient and functional activation of T-cells [75]. Signaling by the chimeric receptor with one of these signaling domains occurs most probably via similar cascades as does endogenous TCR activation and results in cytolytic activity and secretion of cytokines like IL2, IFN- $\gamma$ and TNF- $\alpha$.

Adding a co-stimulatory signal might provide an advantage for optimal and persistent functioning of T-cells, their development into memory cells and re-activation, especially by targets lacking the ligands for co-stimulatory molecules such as tumor cells. For this matter, groups have designed tripartite chimeric receptors made of $\mathrm{scFv}$ linked to the intracellular part of co-stimulatory molecules such as CD28, OX40 (CD134), inducible costimulator (ICOS), programmed cell death protein 1 (PD-1) and 4-1BB (CD137) in series with the signaling moiety from the TCR and, thus, could provide both stimulatory and co-stimulatory signals for T-cell activation. Interaction of these different co-stimulatory receptors with their corresponding ligands results in a distinct function, depending on the nature of the stimulus and the antigenic history of a T-cell on which the chimeric receptor is expressed. For example, efficient CD28 signaling is accompanied by the induction of ICOS, which, in turn, co-stimulates $\mathrm{CD}^{+}$ T-cell activation [76]. Activation of OX40 promotes the expression of B cell leukaemia/lymphoma like X $(B c l-X L)$ and $\mathrm{B}$ cell leukaemia/lymphoma 2 ( $B c l-2)$, enhances the number and survival of antigen-specific effector T-cells and generates potent antigen-specific $\mathrm{CD}^{+}$T-cell memory [77]. Studies which compare these co-stimulatory molecules in the context of chimeric receptors showed notably enhanced cytokine release and killing when tripartite chimeric receptors were compared with chimeric receptors that did not contain any costimulatory signaling [78,79]. Finney et al. compared the effect of the co-stimulatory domains of CD28, ICOS, OX40 (CD134) and 4-1BB (CD137) in the context of the CD33specific chimeric receptor in unstimulated human $\mathrm{CD}^{+}$and CD8 ${ }^{+}$T-cells. In this setting expression of domains derived from CD28 or ICOS were beneficial compared with domains derived from CD134 and CD137 in terms of mediating antigen-specific cytokine secretion and lysis of the target cells by T-bodies [78]. The advantage may be tumor- (costimulation) specific, since there appears to be no advantage to the CD134 domain when targeting e.g., $\mathrm{B}$ cell tumors which have costimulatory molecules. In another study, the CD137 signaling domain was used as the co-stimulatory moiety in the tripartite (anti-CD19 4-1BB- $\zeta$ ) chimeric receptor and was found to elicit potent cytotoxicity of T-bodies against acute lymphoblastic leukemia (ALL) cells in-vitro [80]. Moreover, sequential addition of CD28 and CD134 together in the chimeric receptor specific to ganglioside GD2 gives an additional proliferative advantage of T-bodies in response to neuroblastoma cells [81]. 


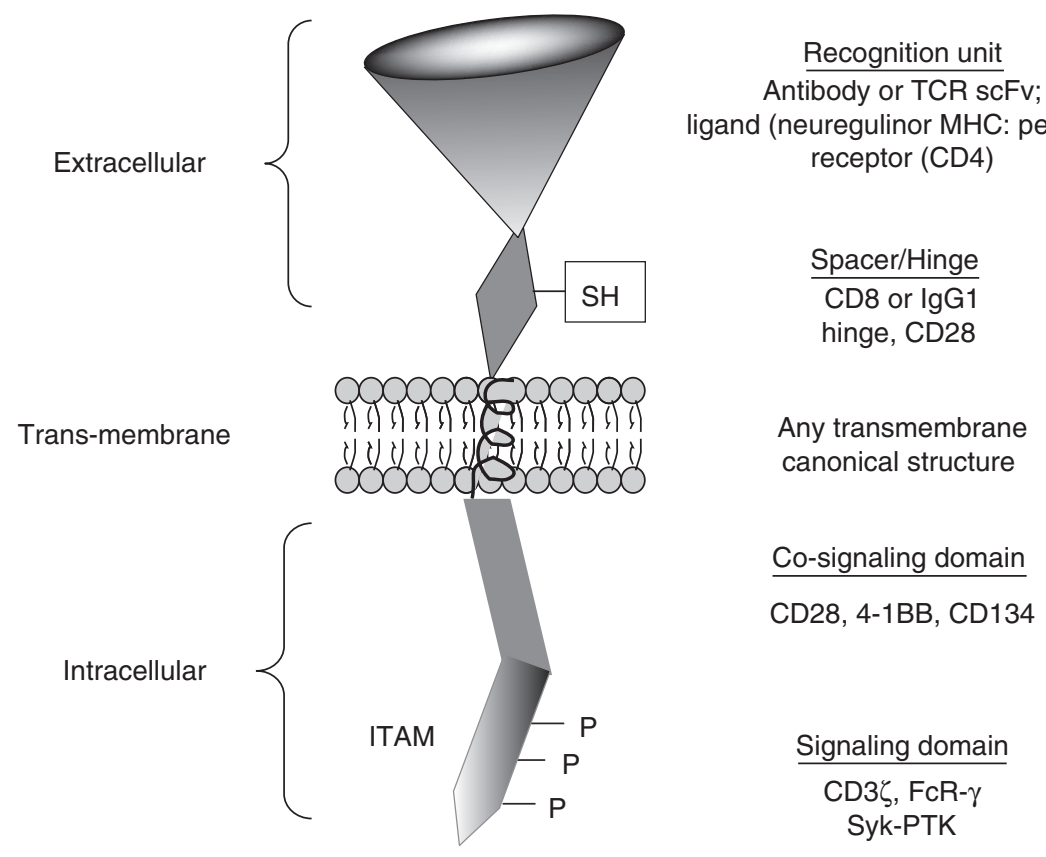

Figure 3. Chimeric receptor design. A schematic presentation of a modular chimeric receptor is shown. The extracellular domain is composed of the recognition unit that can be either scFv of the antibody or TCR or receptor such as CD4 and a hinge that keeps the recognition unit away from the membrane providing flexibility advantageous for recognition. Any canonical transmembrane structure can be used for the transmembrane domain, followed by co-signaling and signaling moieties.

ITAM: immunoreceptor tyrosine-based activation motif; PTK: protein tyrosine kinase; Syk: spleen tyrosine kinase.

Nevertheless, many groups have still chosen to selectively use CD28 as a costimulatory domain in chimeric receptors and showed that scFv-CD28- $\gamma / \zeta$ expressing T-cells efficiently kill tumors in different experimental settings in vitro as well as in vivo [74,82]. Moreover, Fridmann-Morvinski et al showed that expression of the tripartite chimeric receptor but not the CD28-truncated chimeric receptor could rescue activated T-cells from antigen-induced cell death [83]. In addition, recent findings showed that a CD28-domain-containing chimeric receptor enhances the resistance of the T-body to $\mathrm{T}$ regulatory cells [66].

Taken all together, the T-body approach demonstrates that antibody specificity, TCR signaling and co-signaling can be combined in one recombinant receptor, which can efficiently activate anti-tumor responses both in vitro and in vivo. However, it remains to be elucidated whether such an artificial signaling results in a physiological T-cell activation as minor changes in amino acid sequences in, for example, the TCR domain can alter memory T-cell formation [36]. Furthermore, these fusion proteins are potentially immunogenic [21].

\section{Clinical trials}

Table 1 summarizes most of the clinical trials using genetically modified T-cells in cancer patients that have been reported to date. Most of these trials were recently initiated and are still recruiting patients and therefore only some groups have published their observations so far. The main goal of these studies is to test feasibility and safety and to provide a 'proof-of-concept'. A completed Phase I study by Kershaw and colleagues [84] showed that adoptive administration of large numbers of T-bodies against $\alpha$-folate receptor (FR) to ovarian cancer patients is safe, but these cells did not persist for a long time and did not localize to the tumor. Furthermore, half of the tested patients developed an inhibitory factor in their sera that significantly reduced reactivity of gene-modified T-cells to respond against FR-positive targets. This inhibitory activity was neutralized by protein $G$, suggesting that the inhibitor is most probably a human anti-mouse antibody. Another trial reported the development of human anti-mouse antibodies in patients with metastatic renal cell carcinoma that received infusions of T-bodies specific to carboxy-anhydrase-IX (CAIX) [85]. These results support the general idea that such anti-idiotypic responses can alter the therapeutic effect of genetically modified T-cells and strongly suggest considering the usage of human derived domains in the design of chimeric receptors. Though, the main problem of the last study was the development of grade 2 to 4 liver enzyme disturbances after four to five infusions of genetically modified T-cells. Liver biopsies from these patients revealed T-cells infiltrating around the bile ducts and CAIX expression on the bile duct epithelial cells, thus suggested an autoimmune-reaction of adoptively transferred T-cells. The trial was sustained and the protocol was modified to inject a low dose of a cG250 antibody to saturate liver tissue 


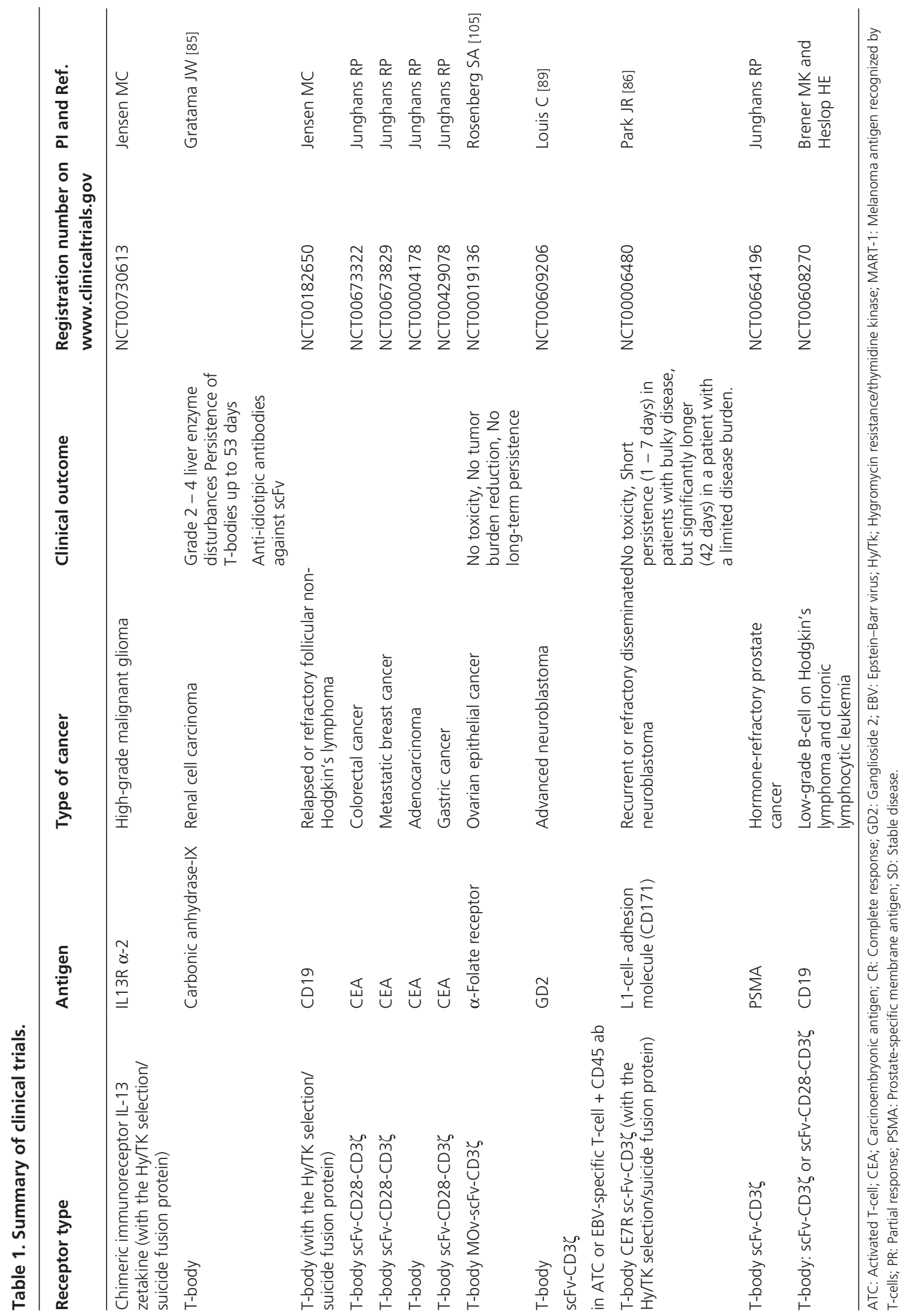




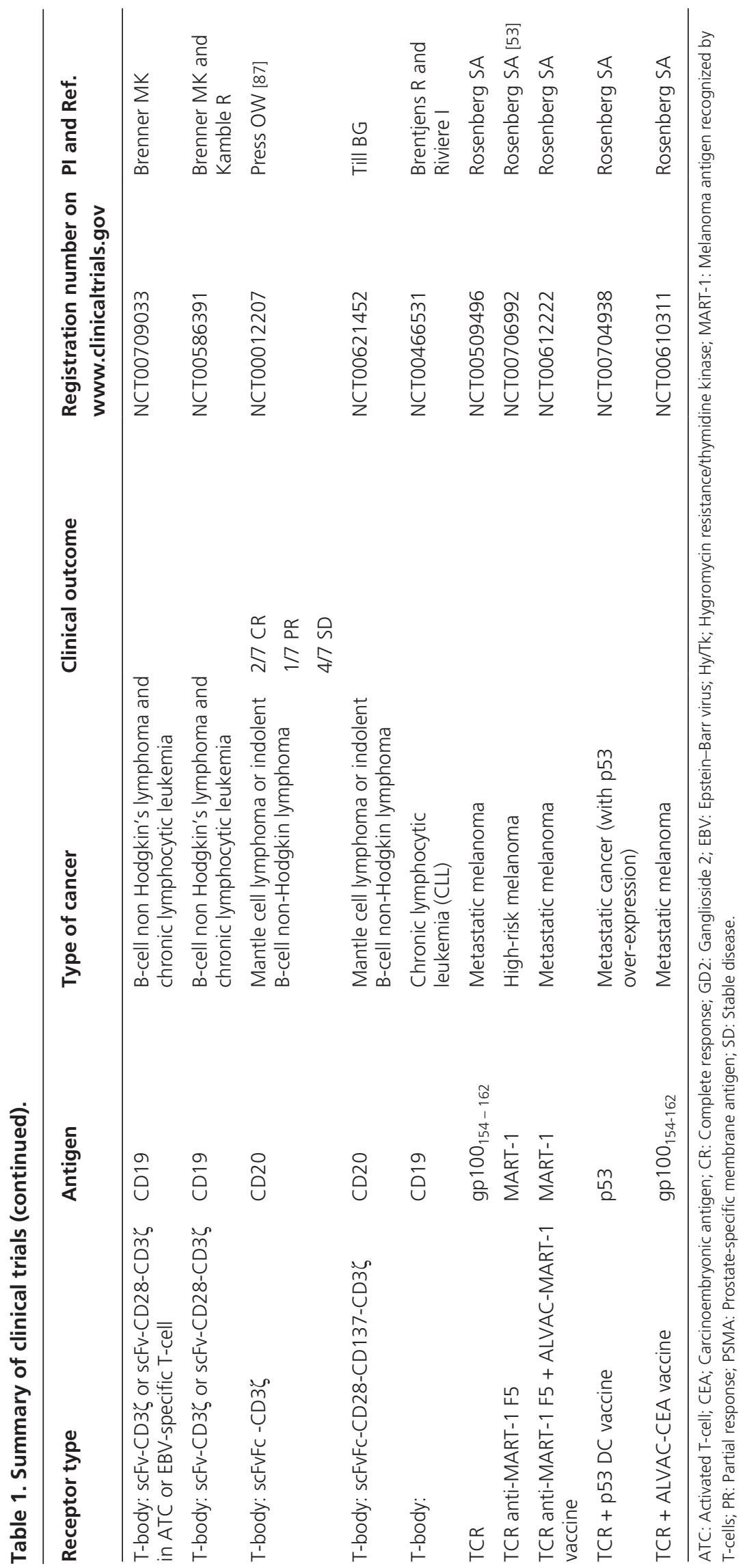


and therefore to protect bile ducts from the damaging effects of scFv (G250)-positive T-cells. Even though, the liver toxicity was not beneficial for treated patients, these observations suggest that injected T-bodies are functional and can react against proteins expressed at the cell surface. Yet, it once again shows the importance of carefully choosing tumorassociated targets for T-cell-mediated immunotherapy and incorporating, for example, suicide genes into adoptively transferred transduced T-cells in order to dampen potential autoimmune responses [48]. In another study using a T-body specific to CD171, an adhesion molecule that is overexpressed in the metastatic neuroblastoma tumors in children, no treatment-associated toxicity was observed [86]. Persistence of genetically-modified T-cells was short in patients with bulky disease, but in patients with limited disease T-cells were detectable for a significantly longer time. However, only one patient experienced prolonged survival following the treatment. A 'proof-of-concept' trial in patients with relapsed or refractory indolent B cell non-Hodgkin's lymphoma and mantle cell lymphoma employed T-bodies against CD20 [87]. Also in this trial T-body administration was well tolerated and not toxic. In patients that received T-cell infusions together with subcutaneous IL2 for 14 days, T-bodies showed a prolonged persistence, compared with T-body administration without IL2. This is in line with the observation that persistence of genetically unmodified T-cell clones can also be improved by the application of IL2 [88] Moreover, six patients received cytoreductive treatment prior to adoptive transfer of T-cells that lead to complete clinical response in two patients and that response was preserved following T-body administration. These observations support the therapeutic potential of genetically modified T-cells for cancer therapy, yet, leaving a big window for improving clinical outcomes.

One possibility for improving anti-tumor activity is to introduce a tumor specific receptor into virus-specific T-cells as e.g., cytomegalovirus (CMV) or Epstein-Barr virus (EBV)specific T-cells in order to improve long term persistence of adoptively transferred T-cells. This is based on the assumption that survival of genetically modified T-cells will be supported by triggering through the endogenous receptor, which is repetitively activated in order to control the CMV or EBV infection. A clinical trial performed in patients suffering from neuroblastoma compared the therapeutic activity of activated T-cells versus EBV-specific T-cells that were transduced with GD2-specific scFv-CD3 $\zeta$ CR [89]. Indeed, EBV-specific T-bodies demonstrated superior persistence in patients when compared with T-bodies without virus specificity. Administration of such virus-specific T-bodies was safe and associated with tumor regression or necrosis in half of the patients tested. These results suggest that combination of tumor-specific receptors and endogenous virus-reactive TCRs improves survival of transferred T-cells and clinical outcome. However, whether the chronic antigen-stimulation can also result in a downmodulation of the introduced receptor [90] or whether chronic antigen-stimulation results in exhaustion or deletion of transferred T-cells [91] remains to be elucidated. However, the observation that gene-marked EBV-specific $\mathrm{T}$ cells have been detected for up to 38 months after infusion [92], suggests that at least EBV-specific T cells do not rapidly become deleted.

One clinical trial has been published so far that transferred TCR-gene-modified T-cells into patients [53]. This clinical proof-of-concept study demonstrates for the first time the safety and feasibility of administering large numbers of TCR-transduced T-cells to cancer patients. Using a strategy developed by this group to take advantage of the host providing homeostatic proliferative signals to transferred cells following lymphodepletion [47,93], the authors demonstrated remarkable long-term in vivo persistence of TCR-transduced T-cells as well as sporadic clinical responses. However, the response rate was well below the expected response rate anticipated from the administration of tumor infiltating lymphocytes (TILs) as reported by this group in similar situations [47,93] and there was no compelling correlation between early/late engraftment of TCR-transduced T-cells and the observed clinical responses.

\section{Expert opinion}

Although first clinical trials demonstrated the feasibility of the transfer of gene-modified T-cells and provide suggestive evidence for efficacy, multiple ongoing and new clinical trials are needed to improve this treatment option. This includes questioning the choice of antigen and the optimal design of the antigen-specific TCR or chimeric receptor in order to improve safety and efficacy of this treatment approach. The ultimate goal remains not only to treat an existing tumor burden but to implement a 'guardian against cancer', the tumor-specific memory T-cell, in order to prevent relapse. In this context which T-cell population should be transduced in order to guarantee memory T-cell formation and whether a large ex vivo expansion of transduced T-cells is necessary prior to application needs to be explored. Furthermore, persistence of genetically modified T-cells could be improved by vaccination strategies.

However, even then, $\alpha \beta$ T-cell based immune-interventions are still hampered by the limited knowledge of antigens/proteins selectively expressed by cancer cells and in particular TCR based strategies are hampered by HLA-restrictions and tumor-escape mechanisms such as HLA-downmodulation, transporter associated with antigen presentation (TAP)-deficiency, or point mutations in the targeted epitope. T-bodies provide an alternative source of receptors to redirect a T-cell in a HLA-independent fashion against cancer cells but provide, due to the genetic engineering of fusion proteins, neo-antigens which can promote rejection of transduced T-cells. Furthermore, it is not clear whether a T-body is a functional fully competent T-cell. Therefore, our laboratory got interested in the ability of $\gamma \delta$ T-cells and their receptors to mediate a broad tumorreactivity. $\gamma \delta$ T-cells have not been subjected to the normal rigors of negative selection. The recent elucidation of recognition of universal MHC-like stress-induced self-antigens such as 
MHC class I polypeptide-related sequence A (MICA) [94], and mevalonate metabolites (phosphoantigens) [95] on tumor cells by $\gamma \delta$ T-cells through the $\gamma \delta$ TCR allows now a rational design of $\gamma \delta$ T-cell or $\gamma \delta$ TCR-based immunotherapies. The potential of $\gamma \delta$ T-cells and their receptors to lyse tumor cells in vitro has been extensively demonstrated (for review see $[96,97])$. However an in vivo or ex vivo activation and expansion of cancer-reactive $\gamma \delta$ T-cells in cancer patients is frequently not feasible as only a subset of patients harbors a fully cancer-reactive $\gamma \delta$ T-cell repertoire that can be expanded [98]. A similar situation has also been described for patients suffering from HIV or Mycobacterium tuberculosis infection [99]. Thus, the availability and capacity to activate and expand antigen-reactive $\gamma \delta$ T-cells in vivo is essential for antigen-clearance but frequently impaired in diseases [98,99].

To overcome limitations of $\alpha \beta$ and $\gamma \delta$ T-cell based strategies, we propose to introduce defined cancer-reactive $\gamma \delta$ TCR into $\alpha \beta$ T-cells in order to redirect T-cells against cancer cells. It has been demonstrated that the transfer of an antigen-reactive $\gamma \delta$ T-cell receptor into a Jurkat-cell line could transfer non-MHC restricted antigen-specificity $[100,101]$. Thus, the transfer of $\gamma \delta$ TCR into $\alpha \beta$ T-cells could be used for reprogramming non-tumor-reactive $\alpha \beta$ T-cells from patients who are devoid of a suitable tumor-reactive T-cell repertoire. This strategy takes advantage of a universal-tumor reactive receptor from a $\gamma \delta$ T-cell and the proliferation potential of $\alpha \beta$ T-cells which is, in contrast to the proliferation capacity of $\gamma \delta$ T-cells [98], still preserved in advanced diseases [53]. This concept would have further advantages: First, in contrast to $\alpha \beta$ TCRs [5] $\gamma \delta$ TCR do not pair with endogenous $\alpha \beta$ TCR chains [102], thus this strategy prevents generation of unwanted specificities. Second, these genetically modified cells should be not immunogeneic as they do not harbor fusion or non-self-proteins as has been reported for multiple genetically modified T-cells [21]. Third, $\gamma \delta$ T-cells and their receptors do not substantially mediate graft-versus-host disease (GvHD) [103] and fourth, the transfer of a $\gamma \delta$ TCR into CMV-reactive T-cells or the transfer of a $\gamma \delta$ TCR with shared reactivity against tumor and CMV infected cells would allow redirection of a T-cell against tumor cells and infection, which is appealing for patients following allogeneic stem cell transplantation [104]. However, it remains to be elucidated whether $\gamma \delta$ TCR transduced $\alpha \beta$ T-cells are immunologically fully competent T-cells in terms of effector, homing and memory function. The other major obstacle to this strategy is that ligands of $\gamma \delta$ TCRs and the exact recognition mechanism need to be better defined.

\section{Acknowledgment}

This work is support by the ZonMW (40-40300-98-07003). We are thankful to the helpful discussions with $\mathrm{Z}$ Eshhar, PD Greenberg and M Theobald.

\section{Declaration of interest}

The authors declare no conflict on interest and have received no payment for the preparation of this manuscript.

\section{Bibliography}

Papers of special note have been highlighted as either of interest $(\bullet)$ or of considerable interest $(\bullet)$ to readers.

1. Renkvist N, Castelli C, Robbins PF, Parmiani G. A listing of human tumor antigens recognized by $\mathrm{T}$ cells.

Cancer Immunol Immunother 2001:50:3-15

2. Singh-Jasuja H, Emmerich NP,

Rammensee HG. The Tubingen approach: identification, selection, and validation of tumor-associated HLA peptides for cancer therapy. Cancer Immunol Immunother 2004; $53: 187-95$

3. Rosenberg SA, Yang JC, Restifo NP. Cancer immunotherapy: moving beyond current vaccines. Nat Med 2004;10:909-15

4. Dudley ME, Rosenberg SA. Adoptive-cell-transfer therapy for the treatment of patients with cancer. Nat Rev Cancer 2003;3:666-75

5. Kuball J, Dossett ML, Wolfl M, et al. Facilitating matched pairing and expression of TCR chains introduced into human T cells. Blood 2007;109:2331-8

6. Schumacher TN. T-cell-receptor gene therapy. Nat Rev Immunol 2002;2:512-9

7. Eshhar Z. The T-body approach: redirecting $T$ cells with antibody specificity. Handb Exp Pharmacol 2008;329-42

8. Clay TM, Custer MC, Sachs J, et al. Efficient transfer of a tumor antigen-reactive TCR to human peripheral blood lymphocytes confers anti-tumor reactivity. J Immunol 1999;163:507-13

9. Willemsen RA, Weijtens ME, Ronteltap C, et al. Grafting primary human $T$ lymphocytes with cancer-specific chimeric single chain and two chain TCR. Gene Ther 2000;7:1369-77

10. Xue SA, Gao L, Hart D, et al. Elimination of human leukemia cells in NOD/SCID mice by WT1-TCR gene-transduced human T cells. Blood 2005;106:3062-7

11. Stanislawski T, Voss RH, Lotz C, et al. Circumventing tolerance to a human
MDM2-derived tumor antigen by TCR gene transfer. Nat Immunol 2001;2:962-70

- This study demonstrates that $\mathrm{T}$ cells can be re-programmed with high-affinity TCRs by circumvention of tolerance.

12. Kuball J, Schmitz FW, Voss RH, et al. Cooperation of human tumor-reactive CD4+ and CD8+ T cells after redirection of their specificity by a high-affinity $\mathrm{p} 53 \mathrm{~A} 2.1$-specific TCR. Immunity 2005;22:117-29

- This study describes the generation of CD8-independent TCRs from HLA-transgenic mice.

13. Holler PD, Holman PO, Shusta EV, et al. In vitro evolution of a $\mathrm{T}$ cell receptor with high affinity for peptide/MHC. Proc Natl Acad Sci USA 2000;97:5387-92

14. Li Y, Moysey R, Molloy PE, et al. Directed evolution of human T-cell receptors with picomolar affinities by phage display. Nat Biotechnol 2005;23:349-54

15. Cohen CJ, Zhao Y, Zheng Z, et al. Enhanced antitumor activity of murine-human hybrid T-cell receptor (TCR) in human lymphocytes is associated 
with improved pairing and TCR/CD3 stability. Cancer Res 2006;66:8878-86

16. Scholten KB, Kramer D, Kueter EW, et al. Codon modification of $\mathrm{T}$ cell receptors allows enhanced functional expression in transgenic human $\mathrm{T}$ cells. Clin Immunol 2006;119:135-45

17. Jorritsma A, Gomez-Eerland R, Dokter M, et al. Selecting highly affine and well-expressed TCRs for gene therapy of melanoma. Blood 2007;110:3564-72

18. Kuball J, Hauptrock B, Malina V, et al. Increasing functional avidity of TCR-redirected $\mathrm{T}$ cells by removing defined $\mathrm{N}$-glycosylation sites in the TCR constant domain. J Exp Med 2009;206:463-75

- This study describes a new method to easily increase the avidity of TCR transduced T-cell by reducing TCR N-glycosylation.

19. Pittet MJ, Rubio-Godoy V, Bioley G, et al. $\alpha 3$ Domain mutants of peptide/ MHC class I multimers allow the selective isolation of high avidity tumor-reactive CD8 T cells. J Immunol 2003;171:1844-9

20. Stauss HJ. Immunotherapy with CTLs restricted by nonself MHC. Immunol Today 1999;20:180-3

21. Berger C, Blau CA, Huang ML, et al. Pharmacologically regulated Fas-mediated death of adoptively transferred $\mathrm{T}$ cells in a nonhuman primate model. Blood 2004;103:1261-9

- This study highlights the immunogeneicity of fusion proteins.

22. Daniels MA, Hogquist KA, Jameson SC. Sweet ' $n$ ' sour: the impact of differential glycosylation on $\mathrm{T}$ cell responses. Nat Immunol 2002;3:903-10

23. Lowe JB. Glycosylation, immunity, and autoimmunity. Cell 2001;104:809-12

24. Moody AM, Chui D, Reche PA, et al. Developmentally regulated glycosylation of the $\mathrm{CD} 8 \alpha \beta$ coreceptor stalk modulates ligand binding. Cell 2001;107:501-12

25. Demetriou M, Granovsky M, Quaggin S, Dennis JW. Negative regulation of T-cell activation and autoimmunity by Mgat $5 \mathrm{~N}$ - glycosylation. Nature 2001;409:733-9

26. Holler PD, Chlewicki LK, Kranz DM TCRs with high affinity for foreign pMHC show self-reactivity. Nat Immunol 2003;4:55-62

- This study shows that high-affinity TCRs engineered by display strategies can become self-reactive.
27. Gao L, Bellantuono I, Elsasser A, et al. Selective elimination of leukemic CD34+ progenitor cells by cytotoxic T lymphocytes specific for WT1. Blood 2000;95:2198-203

28. Theobald M, Biggs J, Hernandez J, et al. Tolerance to $\mathrm{p} 53$ by A2.1-restricted cytotoxic T lymphocytes. J Exp Med 1997;185:833-41

29. Fernandez-Miguel G, Alarcon B, Iglesias A, et al. Multivalent structure of an $\alpha \beta T$ cell receptor. Proc Natl Acad Sci USA 1999;96:1547-52

30. Manolios N, Letourneur F, Bonifacino JS, Klausner RD. Pairwise, cooperative and inhibitory interactions describe the assembly and probable structure of the T-cell antigen receptor. EMBO J 1991; 10:1643-51

31. Rubinstein MP, Kadima AN, Salem ML, et al. Transfer of TCR genes into mature $\mathrm{T}$ cells is accompanied by the maintenance of parental T cell avidity. J Immunol 2003;170:1209-17

32. Cooper LJ, Kalos M, Lewinsohn DA, et al. Transfer of specificity for human immunodeficiency virus type 1 into primary human $\mathrm{T}$ lymphocytes by introduction of T-cell receptor genes. J Virol 2000;74:8207-12

33. Voss RH, Kuball J, Engel R, et al. Redirection of $\mathrm{T}$ cells by delivering a transgenic mouse-derived MDM2 tumor antigen-specific TCR and its humanized derivative is governed by the $\mathrm{CD} 8$ coreceptor and affects natural human TCR expression. Immunol Res 2006;34:67-87

34. Riddell SR, Elliott M, Lewinsohn DA, et al. T-cell mediated rejection of gene-modified HIV-specific cytotoxic $\mathrm{T}$ lymphocytes in HIV-infected patients. Nat Med 1996;2:216-23

35. Sebestyen Z, Schooten E, Sals T, et al. Human TCR that incorporate $\mathrm{CD} 3 \zeta$ induce highly preferred pairing between TCR $\alpha$ and $\beta$ chains following gene transfer. J Immunol 2008;180:7736-46

36. Teixeiro E, Daniels MA, Hamilton SE, et al. Different $T$ cell receptor signals determine CD8+ memory versus effector development. Science 2009;323:502-5

- This study outlines the potential harm of altering the signaling domain in TCRs: a reduced $\mathrm{T}$ cell memory formation.

37. Garboczi DN, Ghosh P, Utz U, et al. Structure of the complex between human T-cell receptor, viral peptide and HLA-A2. Nature 1996;384:134-41
38. Sun ZJ, Kim KS, Wagner G, Reinherz EL. Mechanisms contributing to $T$ cell receptor signaling and assembly revealed by the solution structure of an ectodomain fragment of the CD3 $\varepsilon \gamma$ heterodimer. Cell 2001;105:913-23

39. Pecorari F, Tissot AC, Pluckthun A. Folding, heterodimeric association and specific peptide recognition of a murine $\alpha \beta$ T-cell receptor expressed in Escherichia coli. J Mol Biol 1999;285:1831-43

40. Li ZG, Wu WP, Manolios N. Structural mutations in the constant region of the T-cell antigen receptor (TCR) $\beta$ chain and their effect on TCR $\alpha$ and $\beta$ chain interaction. Immunology 1996;88:524-30

41. Boulter JM, Glick M, Todorov PT, et al. Stable, soluble T-cell receptor molecules for crystallization and therapeutics. Protein Eng 2003;16:707-11

42. Cohen CJ, Li YF, El-Gamil M, et al. Enhanced antitumor activity of $\mathrm{T}$ cells engineered to express T-cell receptors with a second disulfide bond. Cancer Res 2007;67:3898-903

43. Voss RH, Willemsen RA, Kuball J, et al. Molecular design of the $C \alpha \beta$ interface favors specific pairing of introduced TCR $\alpha \beta$ in human T cells. J Immunol 2008;180:391-401

44. Heemskerk MH, Hagedoorn RS, van der Hoorn MA, et al. Efficiency of T-cell receptor expression in dual-specific $\mathrm{T}$ cells is controlled by the intrinsic qualities of the TCR chains within the TCR-CD3 complex. Blood 2007;109:235-43

- This study demonstrates that variable TCR chains have intrinsic qualities to promote pairing.

45. Richman SA, Aggen DH, Dossett ML, et al. Structural features of $\mathrm{T}$ cell receptor variable regions that enhance domain stability and enable expression as single-chain $\mathrm{V} \alpha \mathrm{V} \beta$ fragments. Mol Immunol 2009;46:902-16

46. Blattman JN, Grayson JM, Wherry EJ, et al. Therapeutic use of IL-2 to enhance antiviral T-cell responses in vivo. Nat Med 2003;9:540-7

47. Dudley M, Wunderlich J, Robbins P, et al. Cancer regression and autoimmunity in patients after clonal repopulation with antitumor lymphocytes. Science 2002;298:850-4

- This study demonstrates the efficacy of an adoptive immunotherapy with genetically unmodified T-cells in men. 
48. Berger C, Jensen MC, Lansdorp PM, et al. Adoptive transfer of effector CD8+ T cells derived from central memory cells establishes persistent $\mathrm{T}$ cell memory in primates. J Clin Invest 2008;118:294-305

- This study provides a rationale for transducing T-cells derived from central memory T-cells in order to achieve long term persistence of transferred T-cells.

49. Kaneko S, Mastaglio S, Bondanza A, et al. IL-7 and IL-15 allow the generation of suicide gene-modified alloreactive self-renewing central memory human T lymphocytes. Blood 2009;113:1006-15

50. Leen AM, Myers GD, Sili U, et al. Monoculture-derived T lymphocytes specific for multiple viruses expand and produce clinically relevant effects in immunocompromised individuals. Nat Med 2006;12:1160-6

51. Turk MJ, Guevara-Patino JA, Rizzuto GA, et al. Concomitant tumor immunity to a poorly immunogenic melanoma is prevented by regulatory $\mathrm{T}$ cells. J Exp Med 2004;200:771-82

52. Gattinoni L, Finkelstein SE, Klebanoff CA, et al. Removal of homeostatic cytokine sinks by lymphodepletion enhances the efficacy of adoptively transferred tumor-specific CD8+ T cells. J Exp Med 2005;202:907-12

53. Morgan RA, Dudley ME, Wunderlich JR, et al. Cancer regression in patients after transfer of genetically engineered lymphocytes. Science 2006;314:126-9

54. Backstrom BT, Milia E, Peter A, et al. A motif within the $T$ cell receptor $\alpha$ chain constant region connecting peptide domain controls antigen responsiveness. Immunity 1996;5:437-47

55. Teixeiro E, Daniels MA, Hausmann B, et al. $T$ cell division and death are segregated by mutation of TCR $\beta$ chain constant domains.

Immunity 2004;21:515-26

56. Sahuquillo AG, Roumier A, Teixeiro E, et al. T cell receptor (TCR) engagement in apoptosis-defective, but interleukin 2 (IL-2)-producing, $\mathrm{T}$ cells results in impaired ZAP70/CD3- $\zeta$ association. J Exp Med 1998;187:1179-92

57. Teixeiro E, Fuentes P, Galocha B, et al. T cell receptor-mediated signal transduction controlled by the $\beta$ chain transmembrane domain: apoptosis-deficient cells display unbalanced mitogen-activated protein kinases activities upon $\mathrm{T}$ cell receptor engagement. J Biol Chem 2002;277:3993-4002

58. Pinthus JH, Waks T, Malina V, et al. Adoptive immunotherapy of prostate cancer bone lesions using redirected effector lymphocytes. J Clin Invest 2004;114:1774-81

59. Pinthus JH, Waks T, Kaufman-Francis K, et al. Immuno-gene therapy of established prostate tumors using chimeric receptor-redirected human lymphocytes. Cancer Res 2003;63:2470-6

60. Moritz D, Wels W, Mattern J, Groner B. Cytotoxic T lymphocytes with a grafted recognition specificity for ERBB2-expressing tumor cells. Proc Natl Acad Sci USA 1994;91:4318-22

61. Altenschmidt U, Klundt E, Groner B. Adoptive transfer of in vitro-targeted, activated $\mathrm{T}$ lymphocytes results in total tumor regression. J Immunol 1997;159:5509-15

62. Mamalaki A, Gritzapis AD, Kretsovali A, et al. In vitro and in vivo antitumor activity of a mouse CTL hybridoma expressing chimeric receptors bearing the single chain Fv from HER-2/neu- specific antibody and the $\gamma$-chain from $F_{c}(\varepsilon)$ RI. Cancer Immunol Immunother 2003;52:513-22

63. Gritzapis AD, Mamalaki A, Kretsovali A, et al. Redirecting mouse $\mathrm{T}$ hybridoma against human breast and ovarian carcinomas: in vivo activity against HER-2/neu expressing cancer cells. Br J Cancer 2003;88:1292-300

64. Brentjens RJ, Santos E, Nikhamin Y, et al. Genetically targeted $\mathrm{T}$ cells eradicate systemic acute lymphoblastic leukemia xenografts. Clin Cancer Res 2007;13:5426-35

65. Brentjens RJ, Latouche JB, Santos E, et al. Eradication of systemic B-cell tumors by genetically targeted human $\mathrm{T}$ lymphocytes co-stimulated by CD80 and interleukin- 15 . Nat Med 2003;9:279-86

66. Loskog A, Giandomenico V, Rossig C, et al. Addition of the CD28 signaling domain to chimeric T-cell receptors enhances chimeric T-cell resistance to $\mathrm{T}$ regulatory cells. Leukemia 2006;20:1819-28

67. Jensen MC, Cooper LJ, Wu AM, et al. Engineered CD20-specific primary human cytotoxic $\mathrm{T}$ lymphocytes for targeting B-cell malignancy. Cytotherapy 2003;5:131-8

68. Jensen M, Tan G, Forman S, et al. CD20 is a molecular target for $\mathrm{scFvFc}: \zeta$ receptor redirected $\mathrm{T}$ cells: implications for cellular immunotherapy of CD20+ malignancy.

Biol Blood Marrow Transplant 1998;4:75-83

69. Jensen $M$, Winkler U, Manzke $\mathrm{O}$, et al. Rapid tumor lysis in a patient with B-cell chronic lymphocytic leukemia and lymphocytosis treated with an anti-CD20 monoclonal antibody (IDEC-C2B8, rituximab). Ann Hematol 1998;77:89-91

70. Mitsuyasu RT, Anton PA, Deeks SG, et al. Prolonged survival and tissue trafficking following adoptive transfer of $\mathrm{CD} 4 \zeta$ gene-modified autologous $\mathrm{CD} 4+$ and $\mathrm{CD} 8+\mathrm{T}$ cells in human immunodeficiency virus-infected subjects. Blood 2000;96:785-93

71. Romeo C, Seed B. Cellular immunity to HIV activated by CD 4 fused to $T$ cell or Fc receptor polypeptides. Cell 1991;64:1037-46

72. Eshhar Z, Waks T, Gross G, Schindler DG. Specific activation and targeting of cytotoxic lymphocytes through chimeric single chains consisting of antibody-binding domains and the $\gamma$ or $\zeta$ subunits of the immunoglobulin and T-cell receptors. Proc Natl Acad Sci USA 1993;90:720-4

73. Gross G, Waks T, Eshhar Z. Expression of immunoglobulin-T-cell receptor chimeric molecules as functional receptors with antibody-type specificity. Proc Natl Acad Sci USA 1989;86:10024-8

74. Schaft N, Lankiewicz B, Drexhage J, et al. $T$ cell re-targeting to EBV antigens following TCR gene transfer: CD28-containing receptors mediate enhanced antigen-specific IFN $\gamma$ production. Int Immunol 2006;18:591-601

75. Fitzer-Attas CJ, Schindler DG, Waks T, Eshhar Z. Direct T cell activation by chimeric single chain $\mathrm{Fv}$-Syk promotes Syk-Cbl association and $\mathrm{Cbl}$ phosphorylation. J Biol Chem 1997;272:8551-7

76. Mesturini R, Nicola S, Chiocchetti A, et al. ICOS cooperates with CD28, IL-2, and IFN $-\gamma$ and modulates activation of human naive CD4+ T cells. Eur J Immunol 2006;36:2601-12

77. Rogers PR, Song J, Gramaglia I, et al. OX 40 promotes $\mathrm{Bcl}-\mathrm{xL}$ and $\mathrm{Bcl}-2$ expression and is essential for long-term survival of CD4 $\mathrm{T}$ cells. Immunity 2001;15:445-55 
78. Finney HM, Akbar AN, Lawson AD. Activation of resting human primary $T$ cells with chimeric receptors: costimulation from $\mathrm{CD} 28$, inducible costimulator, CD134, and CD137 in series with signals from the TCR $\zeta$ chain. J Immunol 2004;172:104-13

79. Finney HM, Lawson AD, Bebbington CR, Weir AN. Chimeric receptors providing both primary and costimulatory signaling in $\mathrm{T}$ cells from a single gene product. J Immunol 1998;161:2791-7

80. Imai $\mathrm{C}$, Mihara $\mathrm{K}$, Andreansky M, et al. Chimeric receptors with 4-1BB signaling capacity provoke potent cytotoxicity against acute lymphoblastic leukemia. Leukemia 2004;18:676-84

81. Pule MA, Straathof KC, Dotti G, et al. A chimeric $\mathrm{T}$ cell antigen receptor that augments cytokine release and supports clonal expansion of primary human $\mathrm{T}$ cells. Mol Ther 2005;12:933-41

82. Eshhar Z, Waks T, Bendavid A, Schindler DG. Functional expression of chimeric receptor genes in human $\mathrm{T}$ cells. J Immunol Methods 2001;248:67-76

83. Friedmann-Morvinski D, Bendavid A, Waks $\mathrm{T}$, et al. Redirected primary $\mathrm{T}$ cells harboring a chimeric receptor require costimulation for their antigen-specific activation. Blood 2005;105:3087-93

84. Kershaw MH, Westwood JA, Parker LL, et al. A phase I study on adoptive immunotherapy using gene-modified $\mathrm{T}$ cells for ovarian cancer. Clin Cancer Res 2006;12:6106-15

85. Lamers $\mathrm{CH}$, Sleijfer $\mathrm{S}$, Vulto AG, et al. Treatment of metastatic renal cell carcinoma with autologous T-lymphocytes genetically retargeted against carbonic anhydrase IX: first clinical experience. J Clin Oncol 2006;24:e20-e22

86. Park JR, Digiusto DL, Slovak M, et al. Adoptive transfer of chimeric antigen receptor re-directed cytolytic T lymphocyte clones in patients with neuroblastoma. Mol Ther 2007;15:825-33

87. Till BG, Jensen MC, Wang J, et al. Adoptive immunotherapy for indolent non-Hodgkin lymphoma and mantle cell lymphoma using genetically modified autologous CD20-specific $\mathrm{T}$ cells. Blood 2008;112:2261-71

88. Yee C, Thompson JA, Byrd D, et al. Adoptive $\mathrm{T}$ cell therapy using antigen-specific $\mathrm{CD} 8+\mathrm{T}$ cell clones for the treatment of patients with metastatic melanoma: In vivo persistence, migration, and antitumor effect of transferred T cells. PNAS 2002;99:16168-16173

89. Pule MA, Savoldo B, Myers GD, et al. Virus-specific $T$ cells engineered to coexpress tumor-specific receptors: persistence and antitumor activity in individuals with neuroblastoma.

Nat Med 2008;14:1264-70

- This study demonstrates that transduction of virus-reactive T-cells with tumor-specific receptors can improve survival of transferred T-cells.

90. Heemskerk MH, Hoogeboom M, Hagedoorn R, et al. Reprogramming of virus-specific $T$ cells into leukemia-reactive $\mathrm{T}$ cells using $\mathrm{T}$ cell receptor gene transfer. J Exp Med 2004;199:885-94

91. Barber DL, Wherry EJ, Masopust D, et al. Restoring function in exhausted CD8 $\mathrm{T}$ cells during chronic viral infection. Nature 2006;439:682-7

92. Rooney CM, Smith CA, Ng CY, et al. Infusion of cytotoxic $\mathrm{T}$ cells for the prevention and treatment of Epstein-Barr virus-induced lymphoma in allogeneic transplant recipients. Blood 1998;92:1549-55

93. Dudley ME, Wunderlich JR, Yang JC, et al. Adoptive cell transfer therapy following non-myeloablative but lymphodepleting chemotherapy for the treatment of patients with refractory metastatic melanoma. J Clin Oncol 2005;23:2346-57

94. Groh V, Steinle A, Bauer S, Spies T. Recognition of stress-induced MHC molecules by intestinal epithelial $\gamma \delta$ T cells. Science 1998;279:1737-40

- This study outlines a recognition mechanism of tumors by $\gamma \delta$ T-cells.

95. Gober HJ, Kistowska M, Angman L, et al. Human $T$ cell receptor $\gamma \delta$ cells recognize endogenous mevalonate metabolites in tumor cells. J Exp Med 2003;197:163-8

96. Ferrarini M, Ferrero E, Dagna L, et al. Human $\gamma \delta$ T cells: a nonredundant system in the immune-surveillance against cancer. Trends Immunol 2002;23:14-8

97. Lamb LS Jr, Lopez RD. $\gamma \delta$ T cells: a new frontier for immunotherapy? Biol Blood Marrow Transplant 2005;11:161-8
98. Wilhelm M, Kunzmann V, Eckstein S, et al. $\gamma \delta \mathrm{T}$ cells for immune therapy of patients with lymphoid malignancies. Blood 2003;102:200-6

99. Poccia F, Boullier $\mathrm{S}$, Lecoeur $\mathrm{H}$, et al. Peripheral V gamma 9/V delta $2 \mathrm{~T}$ cell deletion and anergy to nonpeptidic mycobacterial antigens in asymptomatic HIV-1-infected persons. J Immunol 1996;157:449-61

100. Loh EY, Wang M, Bartkowiak J, et al. Gene transfer studies of $\mathrm{T}$ cell receptor-gamma delta recognition. Specificity for staphylococcal enterotoxin A is conveyed by $\mathrm{V}$ gamma 9 alone. J Immunol 1994;152:3324-32

101. Bukowski JF, Morita CT, Tanaka Y, et al. V gamma $2 \mathrm{~V}$ delta 2 TCR-dependent recognition of non-peptide antigens and Daudi cells analyzed by TCR gene transfer. J Immunol 1995;154:998-1006

102. van der Veken LT, Hagedoorn RS, van Loenen MM, et al. $\alpha \beta$ T-cell receptor engineered $\gamma \delta \mathrm{T}$ cells mediate effective antileukemic reactivity. Cancer Res 2006;66:3331-7

103. Anderson BE, Shlomchik WD, Shlomchik MJ. Recipient $\gamma \delta$ T cells in graft-versus-host disease. Blood 2006;107:3808-9

104. Halary F, Pitard V, Dlubek D, et al. Shared reactivity of $\mathrm{V} \delta 2 \mathrm{neg} \gamma \delta \mathrm{T}$ cells against cytomegalovirus-infected cells and tumor intestinal epithelial cells. J Exp Med 2005;201:1567-78

105. Kershaw MH, Westwood JA, Parker LL, et al. A phase I study on adoptive immunotherapy using gene-modified $\mathrm{T}$ cells for ovarian cancer. Clin Cancer Res 2006;12:6106-15

\section{Affiliation}

Victoria Marcu-Malina ${ }^{1}$, Suzanne van Dorp ${ }^{2} \&$ Jürgen Kuball ${ }^{\dagger 1,2}$

${ }^{\dagger}$ Author for correspondence

${ }^{1}$ University Medical Center Utrecht,

Department of Hematology and

VanCreveld Clinic,

Huispostnr.: KC02.085.2; Kamer KB.02.043.1, Lundlaan 6, 3584 EA Utrecht,

The Netherlands

Tel: +31 088755 4982; Fax: +31 088755 4305;

E-mail: j.h.e.kuball@umcutrecht.nl

${ }^{2}$ University Medical Center Utrecht,

Department of Immunology,

The Netherlands 
